\title{
Database Validation Plan
}

National Cancer Institute

\section{Source}

National Cancer Institute. Database Validation Plan. NCI Thesaurus. Code C115620.

A proposed method that defines edit checks, the process of edit checks, and validation tests for database specification. 